\title{
Thin films of organic molecular crystals (OMC) possessing type $B$ lattice: spatial structure of dibenzotetraazaannulene film is related to its thickness
}

\author{
B. A. Snopok*, Ya. D. Lampeka \\ * Institute of Semiconductor Physics of NASU, 45, prospect Nauki, 252028 Kiev, Ukraine \\ E-mail: snopok@isp.kiev.ua, Telephone: +380 (44) 26556 26; ' ${ }^{1}$ Phone (Voice\&Fax): +380 (44) 2651827 \\ L.V.Pisarzhevskij Institute of Physical Chemistry, NAS Ukraine, 31 prospect Nauki, Kyiv, 252039, Ukraine
}

\begin{abstract}
Diffractometric and optical measurements showed that at considerable $\left(>200{ }^{\circ} \mathrm{K}\right)$ difference between sublimation temperature and substrate one dibenzotetraazaannulene forms well oriented films structure of which depends on their thickness. This phenomena was explained by assuming that the temperature gradient inside the film can be changed during the evaporation. Therewith, both diffusion processes in clusters and the restructurization of small crystallites were suggested to be important components of $\mathrm{OMC}$ film formation mechanism.
\end{abstract}

Keywords: molecular crystals, thin films, growth mechanism, organic semiconductors, thermovacuum deposition, layered solids, type B lattice.

Paper received 28.04.99; revised manuscript received 09.07.99; accepted for publication 12.07.99.

\section{Introduction}

Considerable study in the past decades is being given to the mechanisms of formation of semiconductor surfaces because the search for the ways to control their structure is of great importance. Thin films of organic molecular crystals (OMC) fabricated by means of classical for semiconductor microelectronics thermovacuum deposition seem to be especially interesting. Such objects are perspective materials for development of a new generation of electronic devices [1]. Present work is devoted to studying a thin film structure of a new organic semiconductor, dibenzotetraazaannulene, in an attempt to specify the main features of their formation.

\section{Experimental}

The dibenzotetraazaannulene (Fig.1), 5,14-dihydrodibenzo[b,i] $[1,4,8,11]$ tetraazacyclotetradecine $\left(\mathrm{H}_{2} \mathrm{TAA}\right)$, was synthesized according to [2] and recrystallized twice from dimethylformamide. Films of above composition were fabricated via thermovacuum deposition $\left(c a .5 \cdot 10^{-4} \mathrm{~Pa}\right)$ on melted quartz substrates. The thickness of films $(d)$ was determined using a microinterferometer (MII-4) and an ellipso- meter (LEF-3M). Diffractograms were taken with diffractometer DRON-3M ( $\mathrm{Cu} \mathrm{K} \alpha$ radiation, $\lambda=1.54 \AA$ ). The visible reflectance spectra were obtained using spectrophotometer SPECORD-M40 equipped with photometric sphere. The presence of lustre trap allowed to determine both the total reflection coefficient $R$ and its diffuse component $R_{D}$. The mirror component was calculated as $R_{M}=R-R_{D}$. The fractal dimension of films was determined according to [3].

\section{The approximation of stratified structure of thin films}

In the present work the concept of stratified crystal growth [4] is used, in which the normal growth of the crystal is described by consecutive building-on of the layers. In such an approximation some information about growth processes can be obtained from comparative analysis of characteristics of films with different thickness. Therewith, the applicability of this approximation is limited by condition of formation of an ordered structure at least along the stratification direction.

For the study of the film structure along the normal to the substrate, the films were investigated by means of X-ray 


\section{B. A. Snopok, Ya. D. Lampeka: Thin films of organic molecular crystals...}
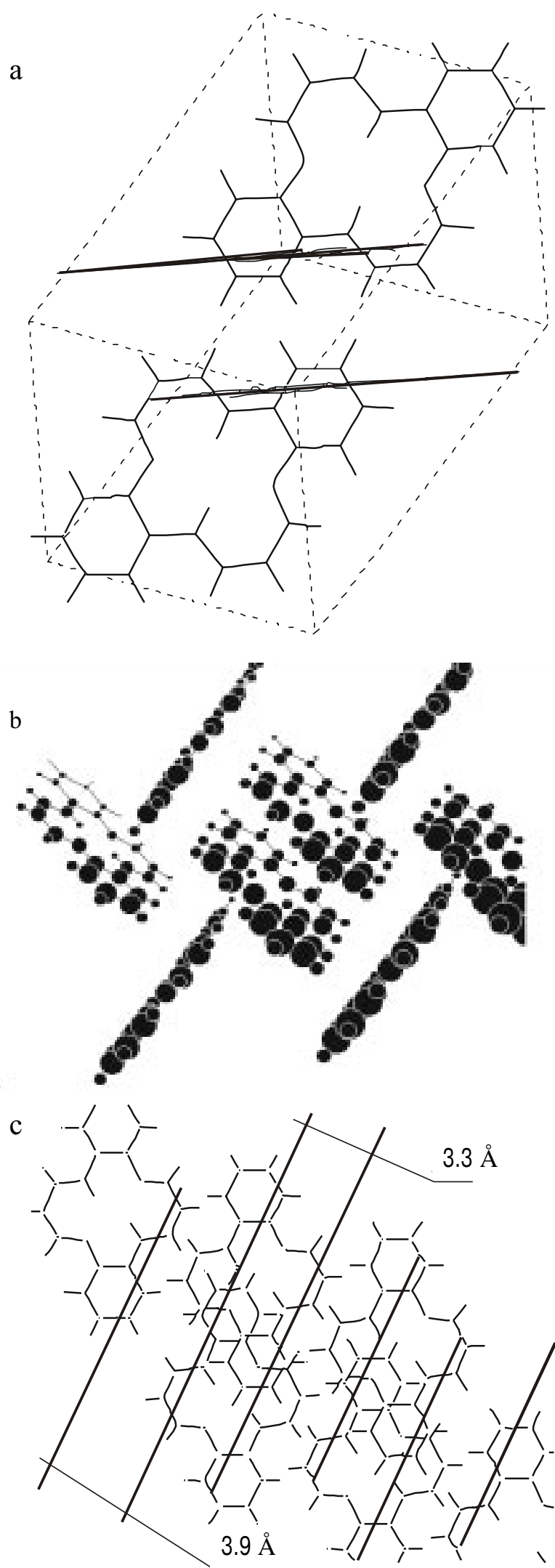

Fig.1. Diagram of the unit cell contents of $\mathrm{H}_{2}$ TAA (a), view illustrating predimeric pairs of $\mathrm{H}_{2}$ TAA in solid state (b) and projection viewed perpendicular to the ab-face (c) - showing the molecular stacking (reconstruction based on the data [6] using MolDraw). diffractometry (Fig.2). One can see that only two peaks are observed on diffractograms of films, in contrast to more than twenty ones characteristic of polycrystalline powder. This evidences the presence of only two sets of reflecting planes at $4.5\left(19^{\circ} 33^{\prime}\right)$ and $9.1\left(9^{\circ} 41^{\prime}\right) \AA$ in the former case. In fact, such feature indicates the formation of oriented thin films of $\mathrm{H}_{2}$ TAA. This is, apparently, the result of enhanced intermolecular interaction in its crystal, which is typical to the type B crystal lattice [5] with two pairs of parallel molecules in an elementary unit [6] (Fig.1).

\section{Origination of crystallization centers.}

Considering that the substance is deposited on the plane amorphous substrate from gaseous phase, one can believe that the adsorption of $\mathrm{H}_{2}$ TAA molecules (process 1, Fig.3) proceeds stochastically. Before the beginning of crystallization, the adlayer molecules diffuse freely along the surface (on the average, during the time $\tau$ with diffusion coefficient $D$, process 3 ). The formation of the solid film begins with spontaneous origination of the complexes of adlayer molecules which lost mobility owing to their collision (process 4). At least for two reasons, the spreading of such complexes along the surface can proceed only owing to the addition of molecules from the adlayer. Firstly, the area of the nuclei is much smaller than the area of substrate. Secondly, the lattice breakage on the boundary of small clusters leads to the enhancement of polarization energy forming in such manner, the repulsive potential which prevent from the adsorption (process 5). The latter effect must also take place on boundaries of large clusters (process 5'). It follows from this, in particular, that all the nuclei have a similar convex shape, comparable dimensions and rates of growth. The average nucleus dimension $\langle r\rangle$ is determined by the diffusion path $\langle l\rangle$ of the adlayer molecules: $\langle r\rangle \approx\langle l\rangle=(4 \cdot D \cdot \tau)^{1 / 2}$. Since both $\tau$ and $D$ depend on temparature, then $\langle r\rangle \sim f(T)$. The last fact is well established for OMC when only the influence of substrate temperature $T_{S}$ is considered [7].

\section{The mechanism of crystal growth}

As it follows from Fig.2, the variations of diffraction peak intensities depending on film thickness indicate the appearance of isolated «pseudomonocrystalline» clusters on early stages of film formation. They are spreaded with the retension of their ordering right up to $d_{\text {crit }} \approx 260 \div 270 \mathrm{~nm}$. It is felt that the substance at this film thickness completely cover the substrate with ordered layer. Further thickening of films leads to formation of stochastically oriented crystallites. It is evident from both the weakening of diffraction peaks and their broadening, as well as from the appearance of new peaks at $2.5,3.4,4.1$ and $6.3 \AA$, typical of polycrystalline sample. The same conclusion can be drawn from optical properties of the films (Fig.4). The increase of diffuse reflectance with thickening films indicates the rise of spatial heterogeneity $\langle l\rangle$ of the samples which is proportional to the average microcrystallite dimensions $\langle r\rangle$.

All above mentioned allows to suggest that after reaching 


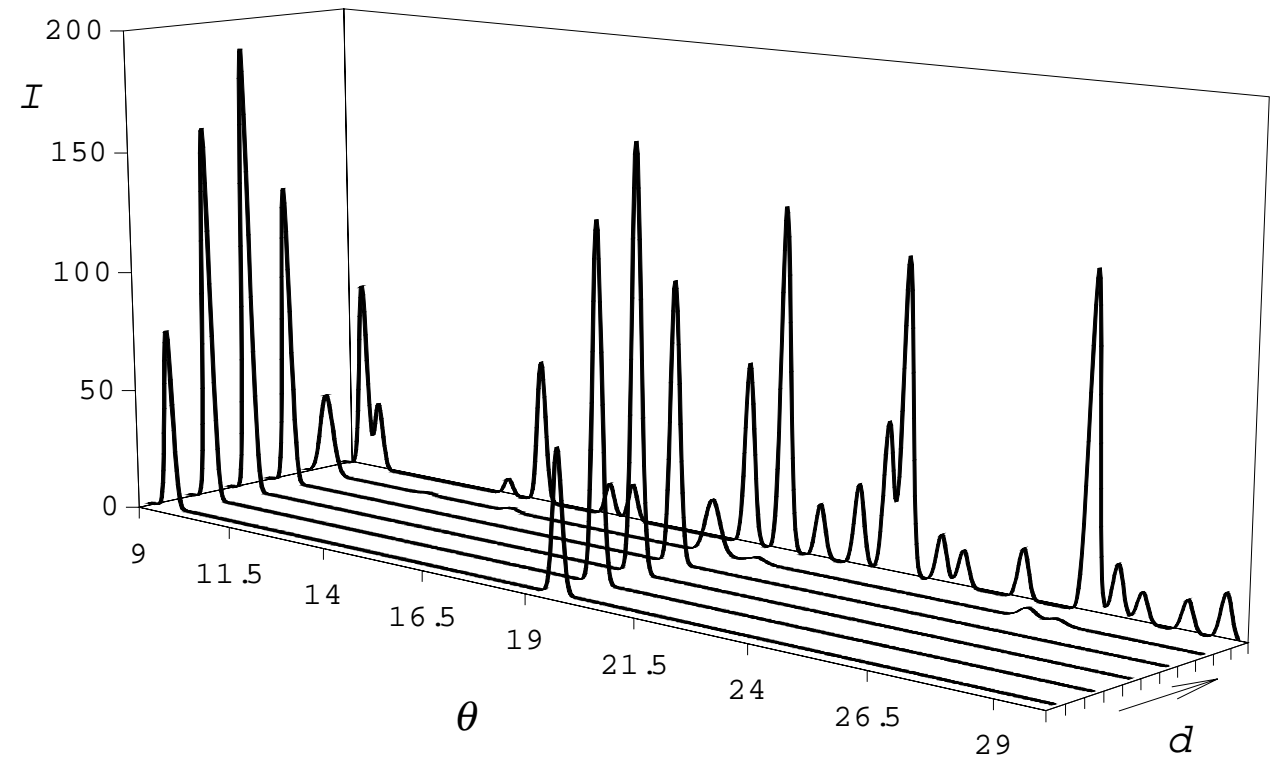

Fig.2. X-ray diffraction patterns of $\mathrm{H}_{2} \mathrm{TAA}$ thin films (rising $d=85,225,260,360,1500 \mathrm{~nm}$, powder).

some definite dimension of surface clusters further growth of the film along the normal to substrate proceeds owing to the adsorption on the nuclei of molecules from gaseous phase (process 1'). In its turn, the adhesion of «hot» molecules can cause the change of temperature distribution inside the film and thereby the increasing $\tau$ in the region of crystallization.

In order to prove this suggestion, the dependence of $T$ vs $d$ was obtained by using a simple model [8]. Let us consider the deposition process in quasistatical approximation when the heat transfer rate by gaseous molecules is much smaller than the heat dissipation rate. The heat transfer by molecules from gaseous phase may be modelled by heat source with the power proportional to $\left(T_{h}-T\right)$ disposed at $x=d\left(T_{h}\right.$ is sublimation temperature). The solution of the equation of heat transfer in such a model at boundary conditions $T=T_{s}(x=0)$ and $T=T_{h}(x=d)$ is:

$\mathrm{T}=\mathrm{T}_{\mathrm{h}}-\frac{\left(T_{S}-T_{h}\right)}{1-\exp (-2 \cdot \beta \cdot d)} \cdot\{\exp (\beta \cdot(x-2 \cdot d))-\exp (-\beta \cdot d)\}$

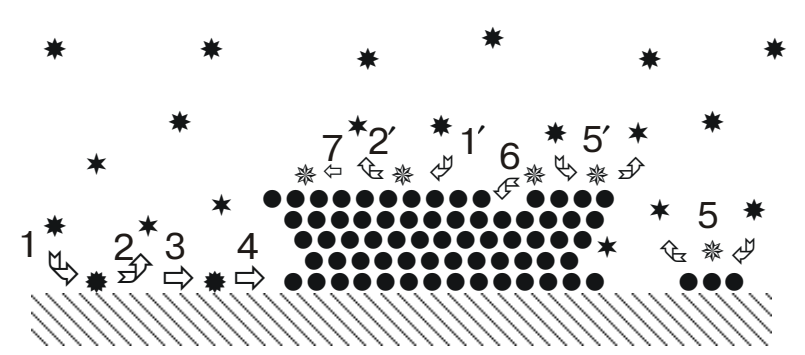

Fig.3. The qualitative model of processes during the growth of films (1, 1'-adsorption, 2, 2'-desorption, 3-diffusion along substratum, 4-complexation, 5, 5'-restructurization, 6-reorganization, 7-diffusion along the cluster; different labels correspond to different energetic and absorption states of molecules). where $\beta$ is some constant. It follows from the dependences of $T v s d$ that the temperature of near the surface layer rises regularly with increasing $d$. Thus, the increase of average crystallite dimensions with thickening the film may be consistently explained by changing temperature gradient inside the film. Since the formation of next layer is possible only on completely crystallized surface, the restructurization of defects (potential well of polarization energy) must be of importance (process 6).

\section{The interaction of crystallites}

Taking into consideration the great difference between the $\mathrm{H}_{2}$ TAA sublimation temperature $(\approx 520 \mathrm{~K})$ and the temperature of substrate $\left(297^{\circ} \mathrm{K}\right)$, the «drop» model of crystallization can not be applied to the system under consideration. We suggest that the contact of two growing crystallites causes the cessation of their growth in contact region but the growth is continued in the rest directions. As a result, the polycrystalline object consisting of the multitude of grains having an irregular structure is formed.

Really, as for the diffusion, at $d<d_{c r i t}$ the splicing of the crystallites promotes much rapid increase of $\langle l\rangle$ (Fig.4) that may be expected if only changing the temperature gradient inside the film is accounted for $(\langle l\rangle \geq\langle r\rangle)$. At $d \approx d_{\text {crit }}$ the condition $\langle l\rangle \approx\langle r\rangle$ is no longer valid since the average diffusion displacement becomes much smaller than the geomertrical dimensions of the sample owing to effective splicing of crystallites. In such a case, diffusion movement leads to the appearance of spatial inhomogeneities with typical dimensions $\langle l\rangle$ and causes formation of disordered structure $(\langle l\rangle \leq\langle r\rangle)$ at $d>d_{\text {crit }}$.

In the above discussion the only intercrystalline interaction taken into consideration was the cessation of the growth of two crystallites in their contact region. In fact, 


\section{B. A. Snopok, Ya. D. Lampeka: Thin films of organic molecular crystals...}

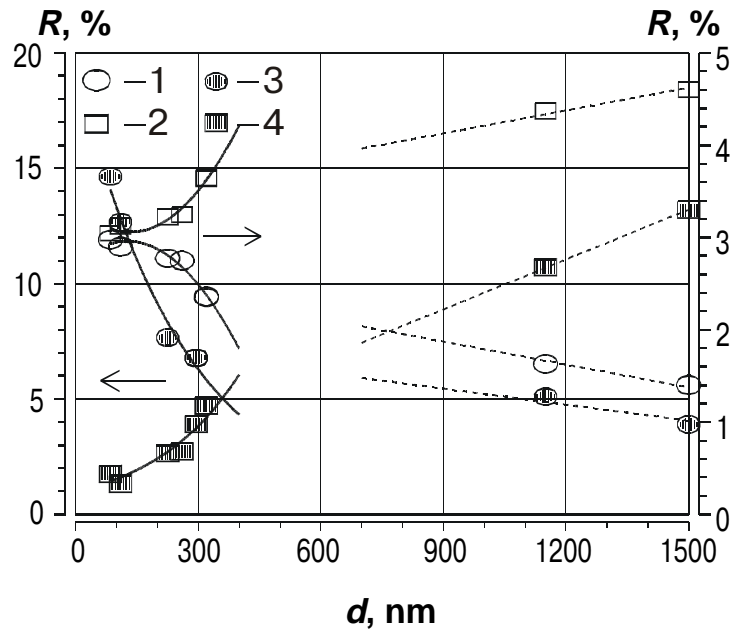

Fig.4. The relationship between mirror $(1,3)$ and diffusion $(2,4)$ reflection in strong $(1,2)$ and weak $(3,4)$ light absorption regions.

however, the picture must be more complicated. The formation of oriented $\mathrm{H}_{2}$ TAA films and the increase of diffuse reflectance with their thickening indicate the stochastic formation of initial nuclei and the development of crystallization front without origination of new nuclei. Physically, this means that small crystallites are reorganized because of their lesser thermodynamic stability [9]. Such a conclusion agrees well with the measurements of film fractal dimensions $D^{H}$. Experimental values of $D^{H}=2 \pm 0.05$ are in good agreement with values resulting from the model of percolation clusters formation in this film [10]. It follows from computer simulations that $D^{H}=2$ can be obtained only when both the reevaporation of small crystallites and the diffusion processes inside clusters are taken into account in the mechanism of film formation $[11,12]$.

\section{References}

1. F. Vogtle. Supramolecular Chemistry, London: John Wiley \& Sons. (1993).

2. H. Hiller, P. Dimroth, H. Pfitzner. 5,14-dihydro-dibenzo[b,i] $[5,9,14,18]$ tetraaza[14]annulen, ein makrocyclischen chelat-bildner. Liebigs. Ann. Chem.,717, pp. 137-147 (1968).

3. C.Allain, M.Cloitre. Fractals in Physics. Proc. 6th Int. Symp., Amsterdam-New York: North-Holland. pp. 91 (1986).

4. V.Z. Belenkiy. Geometrico-probability models of crystallization: phenomenological description, Moscow: Nauka (1980) (in Russian).

5. B. Stevens. Effect of molecular orientation on fluorescence emission and energy transfer in crystalline aromatic hydrocarbons. Spectrochim. Acta., 19, pp. 439-448 (1962).

6. E. Sister, V. Gottfried, M. Kapon, M. Kaftory, Z. Dory, H.B. Gray. Structural characterization of the product of oxidation of a macrocyclic cobalt(II) complex in pyridine solution. Inorg. Chem., 27 (4), pp. 600-604 (1988).

7. M. Yudasaka, M.Kawai, S.Kurita, K. Nakanishi, Y. Kuwae. Thin Solid Films., 151, pp. L115 (1987).

8. B. A. Snopok, Ya. D. Lampeka. About the structure and formation mechanism of thin films of dibenzotetraazaannulene on amorphous substrate. Theoret. and Experim. Chem., 31 (6), pp.365-369 (1995).

9. E. Ruchenstein, B. Nowakowski. Langmuir., 8, pp.1470 (1992).

10. B.A. Snopok, Ya.D. Lampeka. Non-linear electrophysical properties of thin films of dibenzotetraazaannulenes: Application of percolation model. Mol. Cryst. Liq. Cryst., 242, pp.171-177 (1994).

11. P. Meakin, R. Jullien. The growth of self-affine fractal surfaces. Phys. Rev., 41, pp. 983-993 (1990).

12. B.M. Smirnov. Physics of Fractal Aggregates. Moscow: Nauka (1991) (in Russian). 